\title{
ON A MULTICLASS BATCH ARRIVAL RETRIAL QUEUE
}

\author{
G. I. FALIN, ${ }^{*}$ Moscow State University
}

\begin{abstract}
Kulkarni (1986) derived expressions for the expected waiting times for customers of two types who arrive in batches at a single-channel repeated orders queueing system. We propose another method of solving this problem and extend Kulkarni's result to the case of $N \geqq 2$ classes of customers.
\end{abstract}

RETRIALS; BATCH ARRIVALS

In the context of local area computer networks, Kulkarni (1986) considered the following queueing system. There is a single channel, and arriving customers belong to $n$ different types. The arrival times of demands of the $i$ th type ( $i$-demands) form a Poisson process with rate $\lambda_{i}$; at every arrival epoch with certain probability $c_{i k}$ exactly $k i$-demands arrive. These demands we call primary calls. If an arriving batch of $i$-customers finds the channel free, one of the batch members immediately occupies the channel and the rest of the customers in that batch form the sources of repeated $i$-calls ( $i$-sources). Every such source produces a Poisson process of repeated calls with intensity $\mu_{i}>0$. If an incoming repeated call finds a free line it is served and leaves the system after service. Otherwise, if the channel is engaged, the system state does not change. Service times, for primary and for repeated $i$-calls, have the same distribution function $B_{i}(x)$. As usual we suppose that interarrival period, batch sizes, retrial times and service times are mutually independent.

Let $b_{i}(x)=B_{i}^{\prime}(x) /\left(1-B_{i}(x)\right)$ be the instantaneous service intensity of $i$-calls, $\beta_{i}(S)=$ $\int_{0}^{\infty} \exp (-s x) d B_{i}(x)$ be the Laplace-Stieltjes transform of the service time distribution function $B_{i}(x), \beta_{i k}=(-1)^{k} \beta_{i}^{(k)}(0)$ be the $k$ th initial moment of the $i$-calls service time, $c_{i}(z)=\sum_{k=1}^{\infty} c_{i k} z^{k}, \bar{c}_{i}, \sigma_{i}^{2}$ be respectively the generating function, the mean and the variance of batch size of $i$-calls, $\lambda=\lambda_{1}+\cdots+\lambda_{n}, \rho_{i}=\lambda_{i} \bar{c}_{i} \beta_{i 1}$ be the system load due to primary $i$-calls.

$$
\beta(s)=\int_{0}^{\infty} \exp (-s x) d B(x), \quad \beta_{k}(-1)^{k} \beta^{(k)}(0), \quad \rho=\sum_{i=1}^{n} \rho_{i} .
$$

Let $c(t)=0$ if at time $t$ the channel is free; $c(t)=i$ if at time $t$ the channel is occupied by some $i$-call; $N_{i}(t)$ is the number of $i$-sources at time $t$. If $c(t) \neq 0$ then $\xi(t)$ is the time during which the channel has been serving the call which occupies the channel at time $t$.

We shall consider the system in steady state, which exists if and only if $\rho<1$, so the condition $\rho<1$ is assumed to hold from now on. Our goal consists of finding mean queue lengths $N_{i}=E N_{i}(t), \quad 1 \leqq i \leqq n$ as well as the variance-covariance matrix of the $\left(N_{1}(t), \ldots, N_{n}(t)\right)$. Kulkarni (1986) proposed a method of solving the problem and in the case of two types of customers obtained formulas for $N_{1}, N_{2}$. In this note we describe another approach to the problem and obtain a solution in the general case.

Theorem 1 . The expected number of retrial customers of type $i$ in steady state is

$$
N_{i}=\frac{\lambda_{i}\left(\rho+\bar{c}_{i}-1\right)}{\mu_{i}(1-\rho)}+\frac{\lambda_{i} \bar{c}_{i}}{2} x_{i}
$$

Received 22 April 1987; revision received 23 November 1987.

* Postal address: Department of Probability, Mechanics and Mathematics Faculty, Moscow State University, Moscow, 119899, USSR. 
where the values $x_{i}$ can be found as the solution of the system of linear equations

$$
\sum_{j=1}^{n} \frac{\mu_{j} \rho_{j}}{\mu_{i}+\mu_{j}}\left(x_{i}+x_{j}\right)=x_{i}-\sum_{j=1}^{n} \lambda_{j} \bar{c}_{j} \beta_{j 2}-\frac{\beta_{i 1}}{\bar{c}_{i}}\left(\sigma_{i}^{2}+\bar{c}_{i}^{2}-\bar{c}_{i}\right) .
$$

Proof. Let $m=\left(m_{1}, \cdots, m_{n}\right), \quad z=\left(z_{1}, \cdots, z_{n}\right), \quad e_{i}=(0, \cdots, 1, \cdots, 0)$ be the $n$ dimensional vector with $i$ th coordinate equal to 1 and the rest equal to 0 , and $e=(1, \cdots, 1)$ be the $n$-dimensional vector which has all coordinates equal to 1 .

Consider the system in steady state and write:

$$
\begin{gathered}
p_{0}(m)=P\left\{c(t)=0, N_{1}(t)=m_{1}, \cdots, N_{n}(t)=m_{n}\right\} \\
p_{i}(m, x) d x=P\left\{c(t)=i, x<\xi(t)<x+d x, N_{1}(t)=m_{1}, \cdots, N_{n}(t)=m_{n}\right\}, \quad i=1, \cdots, n .
\end{gathered}
$$

In a general way we obtain the equations of statistical equilibrium:

$$
\begin{aligned}
\left(\lambda+\sum_{i=1}^{n} \mu_{i} m_{i}\right) p_{0}(m)= & \sum_{i=1}^{n} \int_{0}^{\infty} p_{i}(m, x) b_{i}(x) d x, \\
\frac{d}{d x} p_{j}(m, x)= & -\left[\lambda+b_{j}(x)\right] p_{j}(x) \\
& +\sum_{i=1}^{n} \lambda_{i} \sum_{k=1}^{m_{i}} c_{i k} p_{j}\left(m-k e_{i}, x\right), \\
p_{j}(m, 0)= & \lambda_{j} \sum_{k=1}^{m_{j}+1} c_{j k} p_{0}\left(m-(k-1) e_{j}\right) \\
& +\mu_{j}\left(m_{j}+1\right) p_{0}\left(m+e_{j}\right) .
\end{aligned}
$$

For the generating functions

$$
\begin{aligned}
\varphi_{0}(z) & =\sum_{m_{1}=0}^{\infty} \cdots \sum_{m_{n}=0}^{\infty} z_{1}^{m_{1}} \cdots z_{n}^{m_{n}} p_{0}(m), \\
\varphi_{i}(z, x) & =\sum_{m_{1}=0}^{\infty} \cdots \sum_{m_{n}=0}^{\infty} z_{1}^{m_{1}} \cdots z_{n}^{m_{n}} p_{i}(m, x)
\end{aligned}
$$

these equations give

$$
\begin{gathered}
\lambda \varphi_{0}(z)+\sum_{i=1}^{n} \mu_{i} z_{i} \frac{\partial \varphi_{0}(z)}{\partial z_{i}}=\sum_{i=1}^{n} \int_{0}^{\infty} \varphi_{i}(z, x) b_{i}(x) d x, \\
\frac{\partial}{\partial x} \varphi_{j}(z, x)=-\left(\sum_{i=1}^{n} \lambda_{i}\left(1-c_{i}\left(z_{i}\right)\right)+b_{j}(x)\right) \varphi_{j}(z, x), \\
\varphi_{j}(z, 0)=\lambda_{j} \frac{c_{j}\left(z_{j}\right)}{z_{j}} \varphi_{0}(z)+\mu_{j} \frac{\partial \varphi_{0}(z)}{\partial z_{j}} .
\end{gathered}
$$

From (2) we find that $\varphi_{j}(z, x)$ depends upon $x$ as follows:

$$
\varphi_{j}(z, x)=\varphi_{j}(z, 0)\left[1-B_{j}(x)\right] \exp (-s x),
$$

where we have denoted $\sum_{i=1}^{n} \lambda_{i}\left(1-c_{i}\left(z_{i}\right)\right)$ by $s$.

From (4) it follows that

$$
\varphi_{j}(z)=\int_{0}^{\infty} \varphi_{j}(z, x) d x=\varphi_{j}(z, 0) \frac{1-\beta_{j}(s)}{s} .
$$


Now with the help of (4) and (5), Equations (1) and (3) can be rewritten as follows:

$$
\begin{aligned}
& \lambda \varphi_{0}(z)+\sum_{i=1}^{n} \mu_{i} z_{i} \frac{\partial \varphi_{0}(z)}{\partial z_{i}}=\sum_{i=1}^{n} \frac{s \beta_{i}(s)}{1-\beta_{i}(s)} \varphi_{i}(z) \\
& \lambda_{j} \frac{c_{j}\left(z_{j}\right)}{z_{j}} \varphi_{0}(z)+\mu_{j} \frac{\partial \varphi_{0}(z)}{\partial z_{j}}=\frac{s}{1-\beta_{j}(s)} \varphi_{j}(z) .
\end{aligned}
$$

In order to find the distribution of the channel state we multiply (7) by $z_{j}$, then sum over $j=1, \cdots, n$ and subtract from (6); after some transformations we get

$$
\varphi_{0}(z)=\sum_{i=1}^{n} \varphi_{i}(z) \frac{\beta_{i}(s)-z_{i}}{1-\beta_{i}(s)}
$$

Fixing some $j$ and putting $z_{i}=1$ for all $i \neq j$,

$$
\varphi_{0}(z)+\sum_{i=1}^{n} \varphi_{i}(z)=\frac{1-z_{j}}{1-\beta_{j}\left(\lambda_{j}-\lambda_{j} c_{j}\left(z_{j}\right)\right)} \varphi_{j}(z)
$$

Setting $z_{j}=1$ and taking into account the normalization condition $\sum_{i=0}^{n} \varphi_{i}(e)=1$ we get

$$
\begin{aligned}
& \varphi_{j}(e)=\rho_{j} \\
& \varphi_{0}(e)=1-\sum_{j=1}^{n} \varphi_{j}(e)=1-\rho .
\end{aligned}
$$

Also, with $z=e(7)$ and (9) yield

$$
\frac{\partial \varphi_{0}(e)}{\partial z_{j}}=\frac{\lambda_{j}\left(\rho-1+\bar{c}_{j}\right)}{\mu_{j}} .
$$

Summing up (7) over $j=1, \cdots, n$ and subtracting from (6) we have

$$
\sum_{i=1}^{n} \lambda_{i}\left[c_{i}\left(z_{i}\right)-\frac{c_{i}\left(z_{i}\right)}{z_{i}}\right] \varphi_{0}(z)+\sum_{i=1}^{n} \mu_{i}\left(z_{i}-1\right) \frac{\partial \varphi_{0}(z)}{\partial z_{i}}=\sum_{i=1}^{n} \lambda_{i}\left(c_{i}\left(z_{i}\right)-1\right) N(z),
$$

where $N(z)=\sum_{i=0}^{n} \varphi_{i}(z)$.

Differentiating (11) with respect to $z_{i} z_{j}$ at the point $z=e$ we obtain, after some algebra,

$$
\begin{aligned}
\left(\mu_{i}+\mu_{j}\right) \frac{\partial^{2} \varphi_{0}(e)}{\partial z_{i} \partial z_{j}}= & \lambda_{i} \bar{c}_{i} N_{j}+\lambda_{j} \bar{c}_{j} N_{i}-\lambda_{i} \lambda_{j}\left(\frac{\rho-1+\bar{c}_{j}}{\mu_{j}}+\frac{\rho-1+\bar{c}_{i}}{\mu_{i}}\right) \\
& +\delta_{i, j} \lambda_{i}\left[\sigma_{i}^{2}+\bar{c}_{i}^{2}-2\left(\bar{c}_{i}-1\right)(1-\rho)-\bar{c}_{i}\right] .
\end{aligned}
$$

Now differentiate (7) with respect to $z_{i}$ at the point $z=e$ :

$$
\begin{aligned}
\frac{1}{\beta_{j 1}} \frac{\partial \varphi_{j}(e)}{\partial z_{i}}= & \mu_{j} \frac{\partial^{2} \varphi_{0}(e)}{\partial z_{i} \partial z_{j}}+\frac{\lambda_{j} \lambda_{i}\left(\rho-1+\bar{c}_{i}\right)}{\mu_{i}}+\lambda_{i} \lambda_{j} \bar{c}_{i} \bar{c}_{j} \frac{\beta_{j 2}}{2 \beta_{j 1}} \\
& +\delta_{i, j} \lambda_{j}\left[c_{j}-1\right)(1-\rho) .
\end{aligned}
$$

Then multiply (13) by $\beta_{j 1}$ and sum up over $j=1, \cdots, n$ :

$$
\begin{aligned}
\sum_{j=1}^{n} \mu_{j} \beta_{j 1} \frac{\partial^{2} \varphi_{0}(e)}{\partial z_{i} \partial z_{j}}= & N_{i}-\frac{\lambda_{i}\left(\rho-1+\bar{c}_{i}\right)}{\mu_{i}}\left(1+\lambda \beta_{1}\right)-\frac{\lambda_{i} \bar{c}_{i}}{2} \sum_{j=1}^{n} \lambda_{j} \bar{c}_{j} \beta_{j 2} \\
& -\lambda_{i} \beta_{i 1}\left(c_{i}-1\right)(1-\rho) .
\end{aligned}
$$


Using (12) we obtain from this equality:

$$
\begin{aligned}
\sum_{j=1}^{n} \frac{\mu_{j} \beta_{j 1}}{\mu_{i}+\mu_{j}}\left(\lambda_{i} \bar{c}_{i} N_{j}+\lambda_{j} \bar{c}_{j} N_{i}\right)= & N_{i}-\frac{\lambda_{i} \bar{c}_{i}}{2} \sum_{j=1}^{n} \lambda_{j} \bar{c}_{j} \beta_{j 2} \\
& -\frac{\lambda_{i} \beta_{i 1}}{2}\left(\sigma_{i}^{2}+\bar{c}_{i}^{2}-\bar{c}_{i}\right)-\frac{\lambda_{i}}{\mu_{i}}\left(\rho-1+\bar{c}_{i}+\lambda \beta_{1} \bar{c}_{i}\right) \\
& +\sum_{j=1}^{n} \mu_{j} \beta_{j 1} \frac{\lambda_{i} \lambda_{j}}{\mu_{i}+\mu_{j}}\left(\frac{\bar{c}_{j}}{\mu_{j}}+\frac{\bar{c}_{i}}{\mu_{i}}\right)
\end{aligned}
$$

Introducing the new variables $x_{i}$ by the formula

$$
N_{i}=\frac{\lambda_{i}\left(\rho+\bar{c}_{i}-1\right)}{\mu_{i}(1-\rho)}+\frac{\lambda_{i} \bar{c}_{i}}{2} x_{i}
$$

completes the proof.

For every concrete $n$ it is easy to obtain the solution in explicit form. For example, if $n=2$ then we have the system of two linear equations with two unknown variables and so after some algebra we get the main results of Kulkarni (1986):

$$
\begin{aligned}
N_{1}= & \frac{\lambda_{1}\left(\rho+\bar{c}_{1}-1\right)}{\mu_{1}(1-\rho)} \\
& +\frac{\lambda_{1} \bar{c}_{1}}{2} \frac{\left[\mu_{2}+(1-\rho) \mu_{1}\right] A+\left[(1-\rho) \mu_{1}+\left(1-\rho_{2}\right) \mu_{2}\right] B_{1}+\mu_{2} \rho_{2} B_{2}}{(1-\rho)\left[\left(1-\rho_{1}\right) \mu_{1}+\left(1-\rho_{2}\right) \mu_{2}\right]} \\
N_{2}= & \frac{\lambda_{2}\left(\rho+\bar{c}_{2}-1\right)}{\mu_{2}(1-\rho)} \\
& +\frac{\lambda_{2} \bar{c}_{2}}{2} \frac{\left[\mu_{1}+(1-\rho) \mu_{2}\right] A+\left[(1-\rho) \mu_{2}+\left(1-\rho_{1}\right) \mu_{1}\right] B_{2}+\mu_{1} \rho_{1} B_{1}}{(1-\rho)\left[\left(1-\rho_{1}\right) \mu_{1}+\left(1-\rho_{2}\right) \mu_{2}\right]}
\end{aligned}
$$

where

$$
A=\sum_{j=1}^{n} \lambda_{j} \bar{c}_{j} \beta_{j 2}, \quad B_{i}=\frac{\beta_{i 1}}{\bar{c}_{i}}\left(\sigma_{i}^{2}+\bar{c}_{i}^{2}-\bar{c}_{i}\right)
$$

It is, of course, generally convenient to use a computer to carry out the calculations.

Our method allows us to obtain second moments of queue lengths

$$
N_{i j}=\frac{\partial N(e)}{\partial z_{i} \partial z_{j}}=E N_{i}(t) N_{j}(t)-\delta_{i, j} \cdot E N_{i}(t) .
$$

For lack of space we consider only the case $c_{i}(z) \equiv z, i=1, \cdots, n$, i.e. singleton arrivals.

Theorem 2 . The second moments of queue lengths in the steady state are

$$
N_{i j}=\lambda_{i} \lambda_{j} x_{i j}+\frac{\lambda_{i} \lambda_{j}}{2} \frac{x_{i}+x_{j}}{\mu_{i}+\mu_{j}}+\frac{\lambda_{i} \lambda_{j}}{\mu_{i} \mu_{j}} \frac{\rho^{2}}{(1-\rho)^{2}},
$$

where the values $x_{i}$ were defined in Theorem 1 and the values $x_{i j}$ can be found as the solution 
of the system of linear equations:

$$
\begin{aligned}
\sum_{k=1}^{n} \mu_{k} \rho_{k} \frac{x_{i j}+x_{i k}+x_{k j}}{\mu_{i}+\mu_{j}+\mu_{k}}= & x_{i j}-\frac{\rho}{2} \frac{x_{i}+x_{j}}{\mu_{i}+\mu_{j}}-\frac{\lambda \beta_{3}}{3} \\
& -\frac{\lambda \beta_{2}}{2} \cdot \frac{\rho}{1-\rho} \cdot \frac{\mu_{i}+\mu_{j}}{\mu_{i} \cdot \mu_{j}} \\
& -\frac{1}{4} \sum_{k=1}^{n} \lambda_{k} \mu_{k} \beta_{k 2}\left(\frac{x_{i}+x_{k}}{\mu_{i}+\mu_{k}}+\frac{x_{j}+x_{k}}{\mu_{j}+\mu_{k}}\right) .
\end{aligned}
$$

The proof is along the lines of Theorem 1, but now we have to differentiate (11) with respect to $z_{i} z_{j} z_{k}$ (instead of differentiating it with respect to $z_{i} z_{j}$ as we did earlier in obtaining (12)) and differentiate (7) with respect to $z_{i} z_{k}$ (instead of differentiating it with respect to $z_{i}$ as we did earlier in obtaining (14)).

\section{References}

KULKARNI, V. G. (1986) Expected waiting times in a multiclass batch arrival retrial queue. J. Appl. Prob. 23, 144-154. 